\title{
TAMAÑO DE CONO Y SEMILLA EN PROCEDENCIAS DE Pinus greggii Engelm. var. greggii ESTABLECIDAS EN DIFERENTES SUELOS
}

\author{
CONE AND SEED SIZE IN PROVENANCES OF Pinus greggii Engelm. var. greggii \\ ESTABLISHED IN DIFFERENT SOILS
}

\author{
Rodrigo Rodríguez Laguna*, Ramón Razo Zárate, Juana Juárez Muñoz, Juan Capulín Grande \\ y Rubén Soto Gutiérrez
}

Instituto de Ciencias Agropecuarias, Universidad Autónoma del Estado de Hidalgo. Rancho Universitario, Avenida Universidad Km 1 s/n. 43600, Tulancingo de Bravo, Hidalgo, México. Tel. directo 01 (775) 7533495 y 01 (771) 7172000 Ext. 4640.

*Autor para correspondencia (rodris71@yahoo.com)

\section{RESUMEN}

La acumulación de materia orgánica en el suelo provoca modificaciones en las características físicas, químicas y biológicas del suelo que afectan el desarrollo, crecimiento y producción de frutos en los árboles. Con el propósito de identificar algunos de estos efectos sobre la producción de semilla en Pinus greggii var. greggii, en este estudio se determinó la variación en tamaño de cono y semilla de nueve procedencias de Pinus greggii var. greggii establecidas en tres tipos de suelo en Galeana, Nuevo León, México. Para ello, en junio de 2010 se colectaron 31 conos cerrados por procedencia por tipo de suelo (837 conos en total) y se midió largo y grueso de cono, y largo, ancho y grueso de semilla. Se encontraron diferencias entre los tipos de suelo $(P \leq 0.01)$ y entre las procedencias $(P \leq 0.01)$, en el tamaño de cono. En el suelo con piedras, $\mathrm{pH}$ moderadamente alcalino y de textura arcillosa, los árboles produjeron conos de menor tamaño $(85.7$ y $36.3 \mathrm{~mm}$ de largo y grueso). En los suelos sin piedras ni caliche, con $\mathrm{pH}$ alcalino y textura arcillo-arenosa, los conos fueron más largos $(89.4 \mathrm{~mm})$ y más gruesos $(37.6 \mathrm{~mm})$. El tipo de suelo no afectó $(\mathrm{P} \leq 0.10)$ el tamaño de semilla, excepto el grosor. En el suelo sin piedras ni caliche, los árboles produjeron semillas ligeramente más gruesas que en el suelo pedregoso (2.0 $\mathrm{mm}$ vs. $1.9 \mathrm{~mm}$ ). La procedencia de Los Lirios, Coahuila produjo los conos más largos (96.1 mm) y en La Tapona, Nuevo León los conos más gruesos $(39.7 \mathrm{~mm})$. Los árboles de Puerto San Juan, Coahuila produjeron la semilla más pequeña, mientras que los de La Tapona produjeron la más grande (3.0 y $2.1 \mathrm{~mm}$ de ancho y grueso).

Palabras clave: Pinus greggii, ensayo de procedencias, tamaño de cono, tamaño de semilla, tipo de suelo.

\section{SUMMARY}

The accumulation of organic matter causes changes on the physical, chemical, and biological characteristics of the soil, which affect tree development and growth, as well as fruit (cone) production. In order to evaluate the effect of soil traits on cone and seed size, nine provenances of Pinus greggii var. greggii were established in three soil types in Galeana, Nuevo León, México. In June 2010, 31 closed cones were collected in each provenance and each soil type (837 cones in total), and their cone length and width, seed length, width and bulk measured. Significant differences between soil types $(P \leq 0.01)$ and between provenances $(P \leq 0.01)$ of $P$. greggii var. greggii were detected in cone size. In the soil with stones, moderately alkaline $\mathrm{pH}$ and clay texture, trees produced smaller cones ( $85.7 \mathrm{~mm}$ long and $36.3 \mathrm{~mm}$ thick), while in the soil without stones or caliche, alkaline $\mathrm{pH}$ and clay-sandy texture, cones were longer $(89.4 \mathrm{~mm})$ and thicker $(37.6 \mathrm{~mm})$. Soil type did not affect $(\mathrm{P} \leq$ 0.10) seed size, except for a slight change on thickness: on soil without stones or caliche, trees produced seeds slightly thicker than in the stony soil $(2.0 \mathrm{~mm} v s .1 .9 \mathrm{~mm})$. The provenance Los Lirios, Coahuila produced the longest cones $(96.1 \mathrm{~mm})$ and La Tapona, Nuevo León formed the thickest cones $(39.7 \mathrm{~mm})$. The trees of Puerto San Juan, Coahuila produced the smallest seed, whereas in La Tapona they produced the largest ones (3.0 and $2.1 \mathrm{~mm}$ of width and thickness).

Index words: Pinus greggii, provenances test, cone size, seed size, soil type.

\section{INTRODUCCIÓN}

El uso de especies nativas en los programas de reforestación es deseable en el país, y para ello se requiere semilla de buena calidad. Sin embargo, la cantidad de semilla producida en un bosque de pino depende del volumen producido el año anterior, del vigor y tamaño del árbol, de las condiciones del clima que hayan prevalecido en el periodo de diferenciación de las yemas florales durante la polinización y maduración de la semilla (Harold y Hocker, 1984), y de las características físicas, químicas y biológicas del suelo (Pritchett, 1991). El tamaño de la copa del árbol refleja la salud y vigor del mismo, ya que un árbol vigoroso produce frutos de mayor tamaño y con mayor número de semillas viables, cuando no es afectado por factores como las fluctuaciones climáticas bruscas, polinización y fertilización inadecuadas, deficiencia de nutrimentos en el suelo, y depredación de conos y semillas por insectos y otros animales.

La materia orgánica desprendida por los árboles contribuye a mantener la fertilidad del suelo e incrementar la productividad de los bosques (FAO, 2000). A medida 
que se acumulan los residuos orgánicos en el suelo también hay mayor proliferación de microorganismos debido al microambiente favorable en el mantillo (Keenan et al., 1996; Martín et al., 1997). La actividad biológica del suelo es considerada altamente sensible a los cambios ambientales, como las fluctuaciones estacionales en humedad, temperatura, $\mathrm{pH}$ y cantidad de materia orgánica (Boerner et al., 2005; Smithwick et al., 2005). La acumulación de reservas nutricionales por el árbol sirven para la producción de frutos.

El suelo es el medio natural que proporciona a los árboles el sostén físico, además de agua y elementos nutricionales para su desarrollo. Con frecuencia estos nutrimentos se encuentran en cantidades insuficientes para satisfacer los requerimientos de los cultivos, o bien están en proporción desbalanceada, lo cual evita que se obtengan los rendimientos máximos potenciales. En ocasiones también ocurre que un elemento nutritivo se encuentra presente en el suelo, pero en una forma que no es aprovechable; es decir, que no es susceptible de ser absorbido por las raíces (Núñez et al., 2010). Aun en una superficie pequeña se pueden encontrar variaciones del suelo, ya que por efecto del clima, la vegetación y la roca de origen, se forman diferentes tipos de suelo con características físicas, químicas y biológicas propias que influyen en el desarrollo y crecimiento de los árboles (Pritchett, 1991).

Pinus greggii Engelm. ocupó el sexto lugar en la producción de planta y reforestación en México en el año 2000 (Musálem y Martínez, 2003), especie en la que se han reportado altas tasas de crecimiento en altura y diámetro en ensayos genéticos o de selección de especies (Castellanos y Ruíz, 1993; López et al., 1999; Rodríguez et al., 2008), así como un alto potencial para adaptarse a condiciones limitantes de humedad (Vargas y Muñoz, 1988). Estas características apoyan el uso de esta especie en programas de reforestación para recuperar suelos degradados en diferentes partes de México, dada sus cualidades de plasticidad fenotípica para crecer en suelos erosionados, pedregosos y poco fértiles (Ramírez et al., 2005).

La ventaja de evaluar un ensayo de procedencias en un mismo clima natural pero con diferentes tipos de suelo permite comparar el comportamiento de semillas provenientes de diferentes poblaciones, con el objeto de valorar los patrones de variación en producción de frutos y semillas, entre otras variables (Zobel y Talbert, 1988).

Debido a la escasa información acerca de la producción de semilla de Pinus greggii var. greggii en diferentes tipos de suelo en la región sur de Nuevo León, en este estudio se planteó determinar la variación en tamaño de cono y semilla de nueve procedencias de Pinus greggii var. greggii establecidas en tres tipos de suelo en el Ejido 18 de Marzo, municipio de Galeana, Nuevo León. Además, encontrar una posible relación entre las variables evaluadas con el origen geográfico de las procedencias.

\section{MATERIALES Y MÉTODOS}

\section{Material biológico}

El estudio se hizo en una plantación de P. greggii var. greggii de 11 años de haber sido plantados, que incluye nueve poblaciones naturales de los Estados de Coahuila y Nuevo León; la ubicación y otras características del sitio de plantación se presentan en el Cuadro 1. La plantación se efectuó en septiembre de 1999 en una parcela del Ejido 18 de Marzo (24 53' LN, $100^{\circ} 12^{\prime} \mathrm{LO}, 2209 \mathrm{~m}$ ), con precipitación media anual de $650 \mathrm{~mm}$ y temperatura media anual de $16{ }^{\circ} \mathrm{C}$. El tipo de clima es $\mathrm{Bs}_{1} \mathrm{kw}$ " (c) que corresponde a un clima seco semi-cálido, con invierno fresco, muy extremoso, con lluvias de verano y precipitación invernal no significativa; los meses más lluviosos en la región son agosto y septiembre (García, 1988).

La plantación se estableció en un terreno agrícola abandonado de forma compacta, con una pendiente promedio de $19 \%$, con exposición noreste, donde hay tres tipos de suelo bien definidos que se identificaron como: (a) suelo con presencia de piedra (95\% de cobertura por piedras ovaladas y redondas), $\mathrm{pH}$ moderadamente alcalino y textura arcillosa; (b) suelo con presencia de caliche, que exhibe $25 \%$ de pedregosidad laminar, $\mathrm{pH}$ alcalino y textura arcillosa; y (c) suelo con ausencia de piedra y caliche, $\mathrm{pH}$ alcalino, textura arcillo-arenosa y alto porcentaje de materia orgánica (Castellanos et al., 2000); en el Cuadro 2 se describen más características físicas y químicas de estos suelos. Debido al uso agrícola anterior, el suelo presenta un nivel de erosión moderado. Al establecer la plantación se hicieron cepas de 30 × 30 × $30 \mathrm{~cm}$, y cada planta se colocó en el centro de la cepa; después se formó un bordo pendiente abajo de la cepa para captar agua de lluvia. Se plantó una hilera de árboles de la misma especie como franja de protección alrededor del área experimental, para controlar el efecto de orilla.

\section{Diseño experimental}

Para considerar las condiciones de cada suelo, así como la pendiente y el componente ambiental en el ensayo, se utilizó un diseño de bloques completos al azar para separar los efectos ambientales de los genéticos en las características de interés (Quijada, 1980). La plantación se dividió en 30 bloques transversales a la pendiente. El suelo con ausencia de piedras y caliche abarcó 16 bloques que representaban $53 \%$ de la superficie, el suelo pedregoso incluyó ocho bloques ( $27 \%$ ), y el suelo con caliche contenía seis bloques 
Cuadro 1. Localización geográfica de las nueve poblaciones naturales de P. greggii var. greggii en el norte del país, utilizadas en el estudio (Ramírez et al., 2005).

\begin{tabular}{lcccc}
\hline \multirow{2}{*}{ Procedencia } & \multicolumn{2}{c}{ Coordenadas geográficas } & \multirow{2}{*}{ Altitud (m) } & \multirow{2}{*}{ Precipitación (mm) } \\
\cline { 2 - 3 } & Lat. Norte & Long. Oeste & & 650 \\
Puerto El Conejo, N. L. & $25^{\circ} 28^{\prime}$ & $100^{\circ} 35^{\prime}$ & 2520 & 650 \\
Santa Anita, Coah. & $25^{\circ} 27^{\prime}$ & $100^{\circ} 34^{\prime}$ & 2560 & 633 \\
Agua Fría, N. L. & $25^{\circ} 26^{\prime}$ & $100^{\circ} 30^{\prime}$ & 2400 & 600 \\
Puerto San Juan, Coah. & $25^{\circ} 25^{\prime}$ & $100^{\circ} 33^{\prime}$ & 2613 & 600 \\
Los Lirios, Coah. & $25^{\circ} 23^{\prime}$ & $100^{\circ} 31^{\prime}$ & 2420 & 500 \\
El Penitente, Coah. & $25^{\circ} 22^{\prime}$ & $100^{\circ} 54^{\prime}$ & 2405 & 600 \\
Jamé, Coah. & $25^{\circ} 21^{\prime}$ & $100^{\circ} 34^{\prime}$ & 2552 & 750 \\
Las Placetas, N. L. & $24^{\circ} 55^{\prime}$ & $100^{\circ} 11^{\prime}$ & 2450 & 650 \\
La Tapona, N. L. & $24^{\circ} 43^{\prime}$ & $100^{\circ} 06^{\prime}$ & 2130 & \\
\hline
\end{tabular}

Cuadro 2. Características físicas y químicas de los tipos de suelo donde se estableció la plantación de P. greggii var. greggii en Galeana, Nuevo León.

\begin{tabular}{|c|c|c|c|c|c|c|c|c|c|c|}
\hline \multirow[t]{2}{*}{ Suelo } & \multicolumn{3}{|c|}{$\begin{array}{l}\text { Proporción de } \\
\text { partículas (\%) }\end{array}$} & \multirow[t]{2}{*}{$\mathrm{T}$} & \multirow[t]{2}{*}{$\mathrm{pH}$} & \multirow[t]{2}{*}{ MO (\%) } & \multirow[t]{2}{*}{$\mathrm{CP}(\%)$} & \multirow[t]{2}{*}{$\mathrm{TP}(\mathrm{cm})$} & \multirow[t]{2}{*}{ FP } & \multirow[t]{2}{*}{ SP (\%) } \\
\hline & Arena & Limo & Arcilla & & & & & & & \\
\hline $\begin{array}{l}\text { Con presen- } \\
\text { cia de piedra }\end{array}$ & 28 & 30 & 42 & Arcillosa & 8.09 & 4.08 & 95 & $0.5-14$ & $\begin{array}{l}\text { Ovaladas y } \\
\text { redondas }\end{array}$ & 27 \\
\hline $\begin{array}{l}\text { Con presen- } \\
\text { cia de caliche }\end{array}$ & 18 & 30 & 52 & Arcillosa & 8.20 & 3.78 & 25 & $12-15$ & Laminar & 20 \\
\hline $\begin{array}{l}\text { Ausencia } \\
\text { de piedra y } \\
\text { caliche }\end{array}$ & 44 & 16 & 40 & Arcillo-Arenosa & 8.24 & 3.26 & 0 & --- & --- & 53 \\
\hline
\end{tabular}

(20\%). Las nueve procedencias se asignaron al azar dentro de cada bloque, en parcelas constituidas por hileras de cuatro plantas a lo largo de la pendiente. Las plantas se establecieron a un espaciamiento de $2 \times 2 \mathrm{~m}$ para generar una densidad de 2500 plantas ha $^{-1}$; hasta el momento de la evaluación no se había modificado la densidad de la plantación, situación que representa alta competencia por luz, humedad y nutrientes entre los árboles.

\section{Muestreo de conos y semillas}

En junio de 2010 se recolectaron 31 conos maduros con semilla, por procedencia y por tipo de suelo, para un total de 837 conos; los conos se colectaron de la parte media de la copa de varios árboles de la misma procedencia, hasta completar la cantidad de conos necesaria para los bloques centrales de cada tipo de suelo, mediante tijeras de mano para podar. Los conos se mantuvieron separados e identificados por procedencia y tipo de suelo. En laboratorio se hicieron las mediciones del largo y grueso de cono cerrado, con un vernier digital marca Mitutoyo ${ }^{\circledR}$ con precisión al milímetro; la longitud se midió desde la base hasta el ápice por la cara interna, y el diámetro se calculó como el promedio de dos medidas perpendiculares entre sí, tomadas en la parte más gruesa del cono, como propusieron Bramlett et al. (1987).

En esa misma fecha se obtuvieron cuatro submuestras por tipo de suelo, con muestreos en zig-zag para abarcar mayor superficie en cada tipo de suelo (Rodríguez y Rodríguez, 2002). Antes de tomar la muestra se limpió la superficie de residuos orgánicos, y con una barrena para suelo de $6 \mathrm{~cm}$ de diámetro se tomó la submuestra de 0 a 30 $\mathrm{cm}$ de profundidad. Las cuatro submuestras se mezclaron para obtener una muestra compuesta por tipo de suelo. Las muestras se llevaron al laboratorio donde se secaron, tamizaron y se sometieron a análisis de textura por el método de Bouyoucos, pH relación 1:2 suelo: agua, y materia orgánica por Walkley-Black (Rodríguez y Rodríguez, 2002). Para determinar la pedregosidad se delimitó $1 \mathrm{~m}^{2}$ en cada tipo de suelo, y de manera visual se estimó la cobertura por piedras en por ciento, y para el tamaño de piedra se midieron varias piedras con una regla graduada en milímetros. 
Los conos se mantuvieron separados por procedencia y se secaron en una estufa de secado marca Grieve ${ }^{\circledR}$ con flujo de aire, durante 48 horas a $60{ }^{\circ} \mathrm{C}$, para que abrieran las escamas y así poder extraer la semilla con ala. Las semillas se desalaron y limpiaron manualmente y se agruparon en dos tamaños, semilla llena (semilla bien desarrollada) y semilla abortiva (semilla no desarrollada, de $1 \mathrm{~mm}$ de largo). Del total de semilla llena obtenida se tomó una muestra al azar de 90 semillas por procedencia y por tipo de suelo, a las que se midió largo, ancho y grueso, con un vernier digital con precisión al milímetro.

\section{Análisis estadístico}

Los valores obtenidos de las variables evaluadas en laboratorio fueron sometidos a un análisis de varianza con el procedimiento GLM del paquete Statistical Analysis System (SAS Institute, 1987); el diseño experimental utilizado fue completamente al azar con arreglo factorial para determinar el efecto de cada uno de los factores y la posible interacción que pudiera darse entre el suelo y las procedencias.

También se hizo comparación de medias con la prueba múltiple de Tukey, con el fin de determinar la mejor combinación procedencia y suelo que pueda favorecer el tamaño de cono y semilla. Con los valores promedio por procedencia se estimó la correlación simple entre las variables medidas directamente en conos y semillas. También se estimó la correlación simple de dichas variables con las coordenadas geográficas y precipitación del sitio de origen del germoplasma, para establecer un posible patrón geográfico de variación.

\section{RESULTADOS Y DISCUSIÓN}

Los árboles de la plantación presentaron una altura de 6.5 $\mathrm{m}$ y un diámetro normal (a $1.30 \mathrm{~m}$ ) de $8.5 \mathrm{~cm}$, en promedio de los tres tipos de suelo. Como hasta el momento de la evaluación no se había modificado la densidad original de la plantación y por las dimensiones morfológicas que han alcanzado los árboles, éstos están en una condición de fuerte competencia por luz, humedad y nutrimentos, pero aun así los árboles dominantes y codominantes son capaces de producir conos con semilla viable. En los árboles que lleguen a la etapa adulta en esta condición, es posible que disminuyan su producción de frutos y semillas viables.

\section{Tamaño de cono}

Se encontraron diferencias significativas $(P \leq 0.01)$ entre procedencias y entre tipos de suelo en el tamaño de los conos (Cuadro 3). Los árboles procedentes de Los Lirios produjeron los conos más largos $(96.1 \mathrm{~mm})$, con $9 \mathrm{~mm}$ por arriba de la media general del ensayo, mientras que los co- nos procedentes de Puerto San Juan alcanzaron $76.8 \mathrm{~mm}$ de largo, con una diferencia de $20 \%$ entre las procedencias extremas. Los árboles de La Tapona tuvieron los conos más gruesos (39.7 mm), y los de Puerto San Juan fueron los más delgados $(34.7 \mathrm{~mm}), 2.3 \mathrm{~mm}$ por debajo de la media general (Figura 1).

El efecto del tipo de suelo sobre el crecimiento del cono se detectó en las nueve procedencias, principalmente en árboles plantados en el suelo con ausencia de piedras y caliche, que produjeron los conos más largos $(89.4 \mathrm{~mm})$, mientras que los más cortos se encontraron en el suelo con presencia de piedras $(85.7 \mathrm{~mm})$, con una diferencia de 3.7 $\mathrm{mm}$ entre los promedios de estas dos condiciones de suelo (Figura 2). Así mismo, se detectó que los árboles en el suelo con presencia de piedras produjeron conos más delgados $(36.3 \mathrm{~mm})$. Los resultados de esta investigación respecto al grueso del cono son similares a los reportados por López et al. (1993), López-Upton y Donahue (1995), y Donahue y López-Upton (1996). El mayor tamaño de conos registrado en el suelo con ausencia de piedra y caliche, se atribuye a la textura que favorece tanto la aireación como la retención de humedad, en beneficio de una buena absorción de agua y nutrimentos (Porta et al., 1999), factores que ayudan al mejor crecimiento y desarrollo de las estructuras vegetativas y reproductivas.

No se encontraron estudios en que se haya evaluado el tamaño de cono en diferente tipos de suelo bajo las mismas condiciones climáticas; sin embargo, hay estudios en los que se han determinado características morfológicas como largo y grueso de cono en poblaciones ubicadas en su distribución natural; al respecto, López et al. (1993) reportaron promedios de 10.5 y $4.0 \mathrm{~cm}$ de largo y grueso de cono respectivamente, en seis poblaciones naturales; estos valores son superiores en $17 \%$ a los aquí encontrados para largo de cono.

Estas diferencias se pueden atribuir a que en el lugar de origen los árboles tienen ya una adaptación a los factores ambientales y han contribuido a reducir el pH del suelo con el aporte y transformación de materia orgánica, y la liberación de ácidos orgánicos que favorecen la disponibilidad y la absorción de los nutrimentos (Pritchett, 1991; Marschner, 1995). Estos efectos se reflejan en varias características morfológicas de la planta (INPOFOS, 1997), y una de ellas es el tamaño del cono. A pesar de que el pH alcalino en los tres tipos de suelo $(>8.1)$ existentes en la plantación del ejido 18 de marzo, y que los valores de $\mathrm{pH}$ reportados en los lugares de origen de las nueve poblaciones varían de 6.0 a 7.4 (Ramírez, 1993; Com. pers. ${ }^{1}$ ), los

\footnotetext{
${ }^{1}$ Ramírez H C (1993) Evaluación de la diversidad genética en poblaciones naturales de Pinus greggii Engelm. Tesis Maestría en Ciencias. Colegio de Postgraduados. Montecillo, México. 90 p.
} 
Cuadro 3. Análisis de varianza y significancia en tamaño de cono y semilla en tres tipos de suelo de nueve procedencias de $P$. greggii var. greggii plantadas en Galeana, Nuevo León.

\begin{tabular}{|c|c|c|c|}
\hline \multirow{2}{*}{ Variables } & \multicolumn{3}{|c|}{ Cuadrados medios } \\
\hline & Procedencias $(8)^{\dagger}$ & Tipo de suelo (2) & Error $(810)^{e}(736)^{e e}$ \\
\hline Largo de cono & $3058.70^{* *}$ & $1115.50^{\star *}$ & 137.20 \\
\hline Grueso de cono & $313.60^{* *}$ & $117.70^{\star *}$ & 14.80 \\
\hline Largo de semilla & $6.49^{* *}$ & $0.60 \mathrm{~ns}$ & 0.35 \\
\hline Ancho de semilla & $0.65^{\star *}$ & $0.17 \mathrm{~ns}$ & 0.09 \\
\hline Grueso de semilla & $0.61^{\star *}$ & $0.65^{\star \star}$ & 0.04 \\
\hline
\end{tabular}
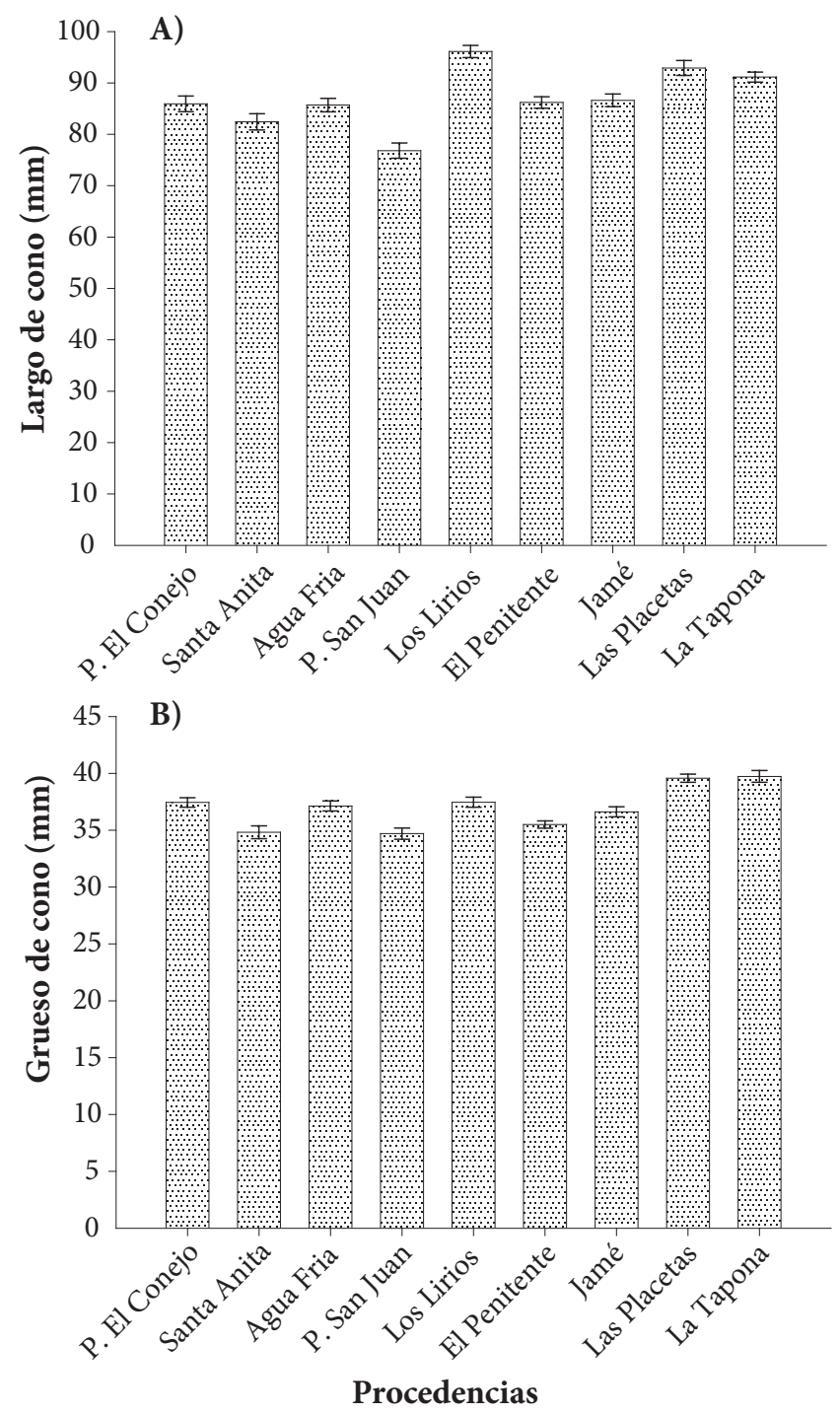

Figura 1. Variación en largo (A) y grueso (B) de cono en nueve procedencias de Pinus greggii var. greggii establecidas en Galeana, Nuevo León. Las líneas sobre las barras indican las desviaciones estándar. 

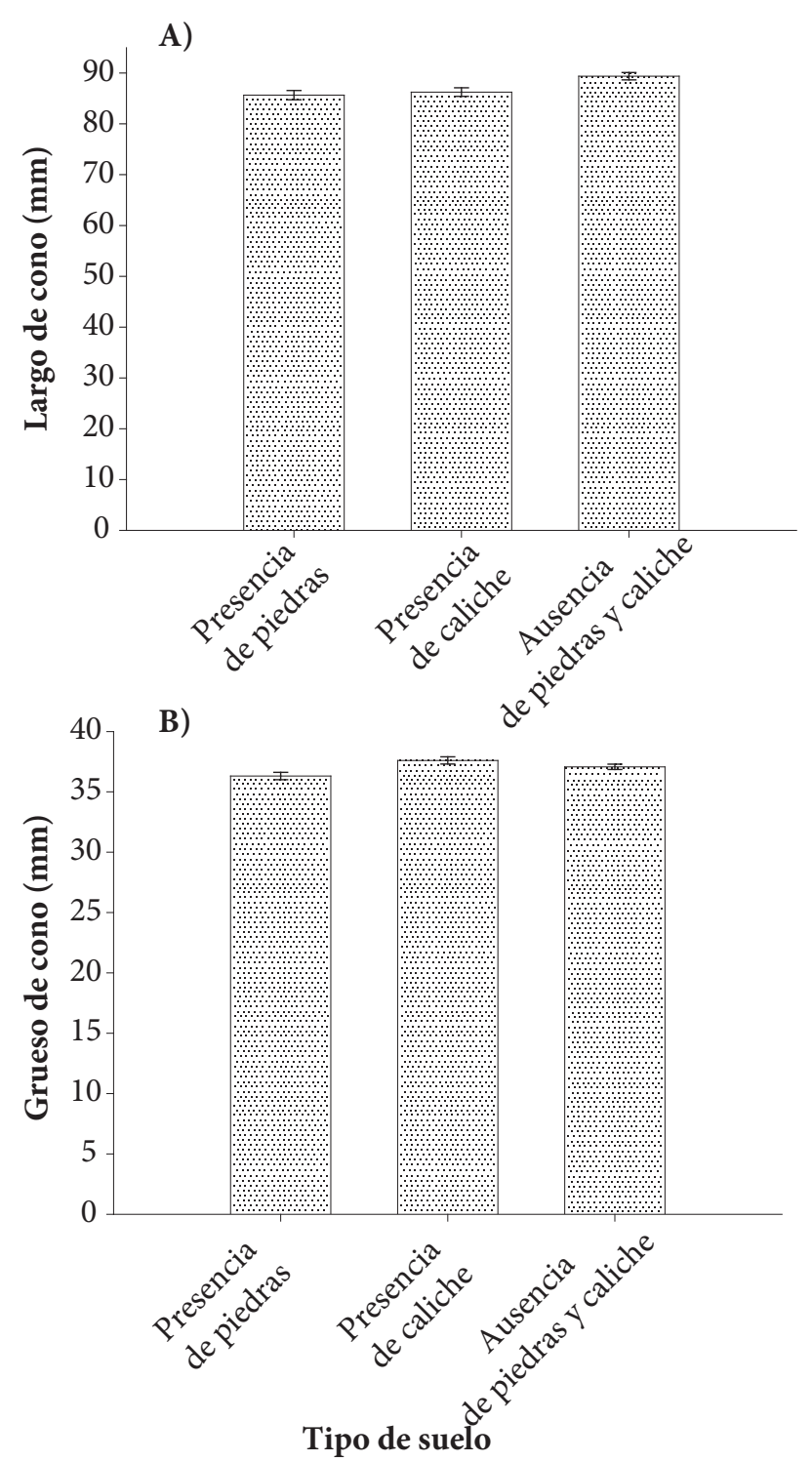

Figura 2. Respuesta del crecimiento en largo (A) y grueso (B) de cono al tipo de suelo en promedio de nueve procedencias de Pinus greggii var. greggii establecidas en Galeana, Nuevo León. Las líneas sobre las barras indican las desviaciones estándar.

árboles de la plantación han podido adaptarse y producir conos aunque más pequeños pero con semilla viable. Este resultado indica la semilla de los árboles de $P$. greggii var. greggii también puede prosperar en suelos alcalinos.

El menor tamaño de los conos en la plantación estudiada se puede deber, por un lado, a que en los suelos con $\mathrm{pH}$ alcalino algunos nutrimentos son menos disponibles para la planta, como el fósforo que interviene directamente en el crecimiento de los conos (INPOFOS, 1997), y por el otro, a la fuerte competencia por nutrimentos en el sistema radical de los árboles por la alta densidad de población, lo que pudo haber provocado una reducción en el crecimiento de las estructuras reproductoras.

\section{Tamaño de semilla}

Los análisis de varianza para largo, ancho y grueso de la semilla mostraron que existen diferencias estadísticas $(\mathrm{P} \leq$ 0.01 ) entre procedencias y en la interacción procedencias $\mathrm{x}$ tipo de suelo (Cuadro 3). El factor tipo de suelo sólo afectó de manera significativa al grueso de la semilla. Los árboles procedentes de Las Placetas produjeron las semillas más largas $(6.4 \mathrm{~mm})$, aunque apenas superaron en 0.3 $\mathrm{mm}$ a la media general del ensayo; en el otro extremo, los árboles de Puerto San Juan produjeron semilla de $5.5 \mathrm{~mm}$ de largo, con una diferencia de $0.9 \mathrm{~mm}$ entre los valores extremos. Para el ancho de semilla los árboles de La Tapona tuvieron el mayor valor ( $3.0 \mathrm{~mm}$ ), y los de Puerto San Juan 
produjeron la semilla más angosta $(2.7 \mathrm{~mm})$, con $0.3 \mathrm{~mm}$ de diferencia entre los valores extremos. Los árboles de La Tapona produjeron las semillas más gruesas $(2.1 \mathrm{~mm})$ que superan en $14.3 \%$ a la semilla producida por los árboles de Los Lirios $(1.8 \mathrm{~mm})$; el resto de las procedencias mostraron valores intermedios (Figura 3 ).

López et al. (1993), Farjon et al. (1997), Donahue y López (1999), Morante et al. (2005) y Alba et al. (2006) han reportado rangos de 5 a $7 \mathrm{~mm}$ en el largo y de $3.0 \mathrm{~mm}$ en el ancho, valores que coinciden con los encontrados en este trabajo. Sin embargo, para el grueso de semilla esos mismos investigadores reportaron $1.9 \mathrm{~mm}$, valor menor al aquí obtenido $(2.1 \mathrm{~mm})$ en semillas producidas por árboles de La Tapona, la procedencia con semillas más gruesas. Se ha postulado que en estas especies un mayor tamaño de semilla favorece la germinación y crecimiento de la plántula, por contar con mayor cantidad de reservas nutritivas para el embrión durante el proceso germinativo (Niembro, 1986), y así ayudar a tener plántulas más robustas.

El tamaño de la semilla y el tipo de suelo sólo mostraron una leve relación con el grueso de la semilla, pues los árboles que crecen en el suelo sin piedras ni caliche produjeron conos con semillas un poco más gruesas $(2.0 \mathrm{~mm})$ que las semillas producidas por los árboles que crecen en suelo pedregoso $(1.9 \mathrm{~mm})$, como se ilustra en la Figura 4 .

La ausencia de asociación relevante entre tamaño de semilla y el tipo de suelo muestra que las diferencias entre suelos afectó poco la formación de semillas en las nueve procedencias estudiadas, aunque hayan afectado el tamaño del cono. La alta densidad de árboles en la plantación y el $\mathrm{pH}$ alcalino del suelo tampoco afectaron el tamaño de semilla, ya que fue comparable al tamaño registrado por otros investigadores sin tales condiciones de estrés.

\section{Patrón geográfico en el tamaño de cono y semilla}

Al correlacionar las variables de tamaño de cono con los datos de origen de las procedencias, se encontró una correlación negativa $(\mathrm{P} \leq 0.05)$ entre el grueso del cono y la latitud, longitud y altitud $(r=-0.80,-0.80$ y -0.69 , respectivamente). Estas correlaciones indican que los árboles que producen conos más gruesos proceden de sitios de menor latitud, longitud y altitud, con un patrón geográfico y altitudinal definido (Cuadro 4). Por otro lado, hubo correlación positiva $(\mathrm{P} \leq 0.05)$ entre el grueso del cono y el largo de la semilla $(r=0.82)$, lo que parece explicable porque los conos más gruesos producen semillas más largas. El resto de las variables evaluadas no presentaron una relación entre ellas. Estos valores son similares a las correlaciones encontradas por López et al. (1993), quienes reportaron que en localidades del norte del país los conos fueron de mayor diámetro y produjeron semilla de mayor longitud, que en localidades del sur.

\section{CONCLUSIONES}

El tipo de suelo afectó significativamente el tamaño de los conos de P. greggii var. greggii, pues los árboles que crecieron en suelo pedregoso, con $\mathrm{pH}$ alcalino (8.1) y de textura arcillosa produjeron conos más pequeños (85.7 y $36.3 \mathrm{~mm}$ de largo y grueso), que los árboles que crecieron en suelos sin piedras ni caliche, con el mismo $\mathrm{pH}$ alcalino y textura arcillo-arenosa $(89.4$ y $39.7 \mathrm{~mm})$. No obstante, el tipo de suelo no afectó significativamente el tamaño de la semilla, excepto el grueso; en suelo con ausencia de piedras y caliche los árboles produjeron semillas ligeramente más gruesas $(2.0 \mathrm{~mm})$ que las producidas en el suelo con presencia de piedras $(1.9 \mathrm{~mm})$.

Hay diferencias significativas entre las nueve procedencias de P. greggii var. greggii en el tamaño (largo y grueso) de los conos. La procedencia de Los Lirios, Coah. produjo los conos más largos $(96.1 \mathrm{~mm})$ y la de La Tapona, N. L. los más gruesos $(39.7 \mathrm{~mm})$. Se encontró un patrón geográfico $y$ altitudinal bien definido en el grueso del cono, pero no en el tamaño de semilla; los árboles que proceden de sitios de menor latitud, longitud y altitud producen conos más gruesos. Además, hubo una correlación positiva entre el grueso del cono y el largo de la semilla. 

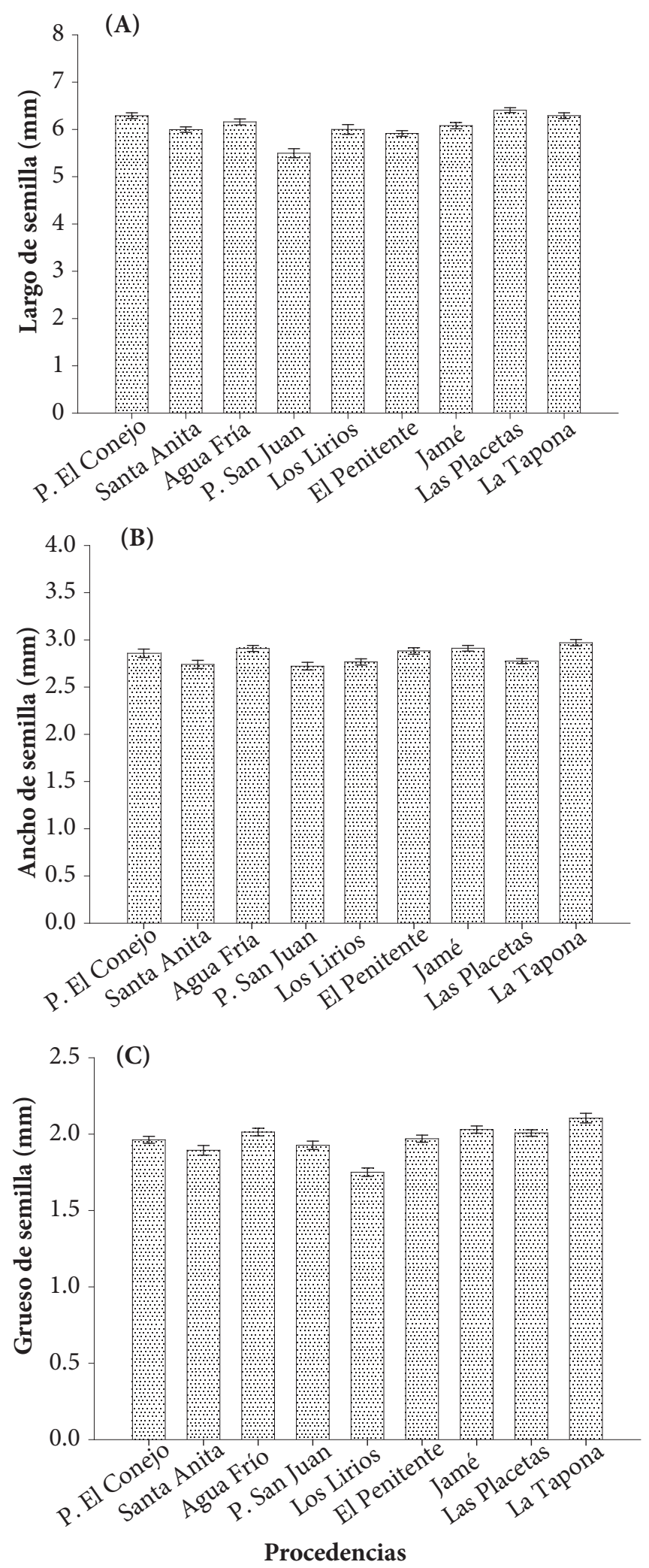

Figura 3. Variación en largo (A), ancho (B) y grueso (C) de semilla en nueve procedencias de Pinus greggii var. greggii establecidas en Galeana, Nuevo León. Las líneas sobre las barras indican las desviaciones estándar. 


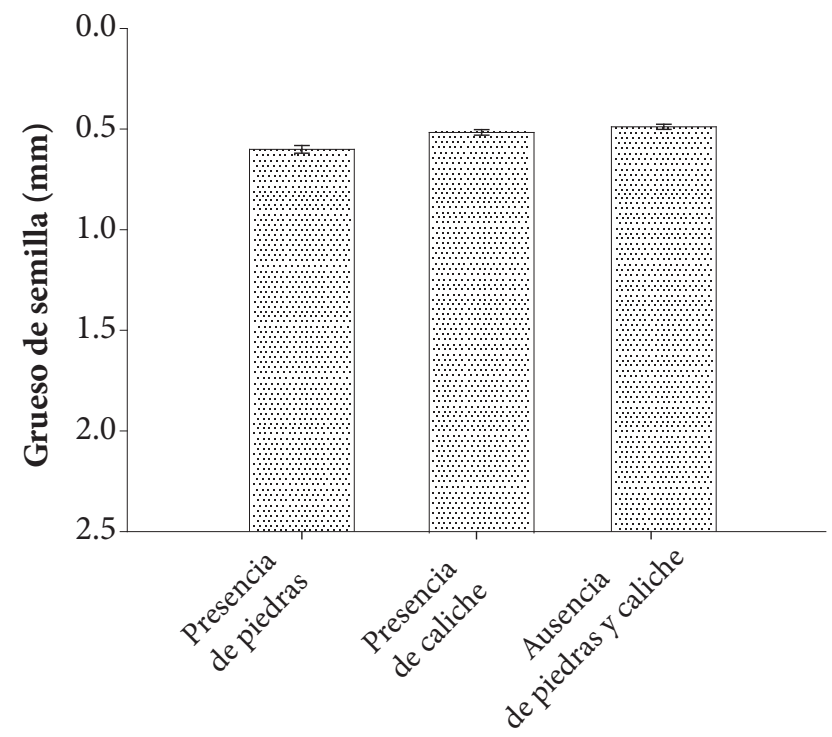

Tipo de suelo

Figura 4. Respuesta en grueso de la semilla al tipo de suelo en las procedencias de Pinus greggii var. greggii establecidas en Galeana, Nuevo León. Las líneas sobre las barras indican las desviaciones estándar.

Cuadro 4. Coeficientes de correlación entre variables geográficas y variables morfológicas de cono y semilla en Pinus greggii var. greggii.

\begin{tabular}{lcccc}
\hline & Ancho de cono & Largo de cono & Largo de semilla & Ancho de semilla \\
\hline \multirow{2}{*}{ Latitud } & -0.80 & -0.51 & -0.52 & -0.37 \\
& $0.009^{\dagger}$ & 0.163 & 0.147 & 0.329 \\
Longitud & -0.80 & -0.43 & -0.54 & -0.15 \\
& 0.009 & 0.249 & 0.133 & 0.699 \\
Altitud & -0.69 & -0.58 & -0.49 & -0.67 \\
& 0.038 & 0.103 & 0.179 & 0.048 \\
\hline
\end{tabular}

†Nivel de significancia de la correlación.

\section{BIBLIOGRAFÍA}

Alba L J, E O Ramírez G, G Rojas P (2006) Variación de semillas de Pinus greggii Engelm. en el municipio de Naolinco, Veracruz, México. For. Veracruzana 8:7-12.

Boerner R E J, J A Brinkman, A Smith (2005) Seasonal variations in enzyme activity and organic carbon in soil of a burned and unburned hardwood forest. Soil Biol. Biochem. 37:1419-1426.

Bramlett D L, E W Belcher, G L DeBar, G D Hertel, R P Karrfalt, C W Lantz, T Miller, K D Ware, H O Yates (1987) Cone Analysis of Southern Pines, a Guidebook. USA. 28 p.

Castellanos B J F, M Ruiz (1993) Introducción de Pinus greggii Engelm. en la Mixteca Alta Oaxaqueña. Folleto de Investigación No. 1. Instituto Nacional de Investigaciones Forestales, SARH. Centro de Investigación Pacífico Sur. Yanhuitlán, Oaxaca, México $18 \mathrm{p}$.

Castellanos J Z, J X Uvalle Bueno, A Aguilar Santelises (2000) Manual de Interpretación de Análisis de Suelos y Agua. Ed. Instituto de Capacitación para la Productividad Agrícola. México. 226 p.

Donahue J K, J López-Upton (1996) Geographic variation in leaf, cone and seed morphology of Pinus greggii Engelm. in native forest. For. Ecol. Manage. 82:145-157.
Donahue J K, J López U (1999) A new variety of Pinus greggii (Pinaceae) in Mexico. Sida 18:1103-1113.

FAO, Food and Agriculture Organization of the United Nations (2000) Global forest resources assessment. (FAO Forestry Paper $N^{\circ}$ 140). Rome, Italy. 357 p.

Farjon A, J A Pérez de la R, B T Styles (1997) Guía de Campo de los Pinos de México y América Central. The Royal Botanic Gardens, Kew-Instituto Forestal de Oxford, Universidad de Oxford. $151 \mathrm{p}$.

García E (1988) Modificaciones al Sistema de Clasificación Climática de Köppen. 4a ed. México, D. F. 219 p.

Harold W, Hocker Jr (1984) Introducción a la Biología Forestal. 1ra. ed. en español. F A Bellomo López (trad). AGT Editor, S. A. México D. F. 446 p.

INPOFOS, Instituto de la Potasa y el Fósforo (1997) Manual Internacional de Fertilidad de Suelos. Ministerio Agropecuario y Forestal. Quito, Ecuador. 141 p.

Keenan R, C Prescott, J Kimmins, J Pastor, B Dewey (1996) Litter decomposition in western red cedar and western hemlock forest on northern Vancouver Island, British Columbia. Can. J. Bot. 74:1626-1634.

López A J L, J J Vargas H, C Ramírez H, J López U (1999) Variación intraespecífica en el patrón de crecimiento del brote terminal de 
Pinus greggii Engelm. Rev. Chapingo S. C. For. Amb. 5:133-140. López U J, J Jasso M, J J Vargas H, J C Ayala (1993) Variación de características morfológicas en conos y semillas de Pinus greggii Engelm. Agrociencia 3:81-95.

Marschner H (1995) Mineral Nutrition of Higher Plants. 2nd ed. Academic Press Limited. London, England. 889 p.

Martín A, J Gallardo, I Santa-Regina (1997) Long-term decomposition process of leaf litter from Quercus pyrenaica forests across a rainfall gradient. Ann. For. Sci. 54:191-202

Morante C J, J Alba L, L del C Mendizábal H (2005) Estudio de conos, semillas y plántulas de Pinus greggii de una población del estado de Veracruz. For. Veracruzana 7:23-31.

Musálem M A, G Martínez C (2003) Monografía de Pinus greggii Engelm. Ed. Jiménez S. A. de C. V. Chapingo, México. 339 p.

Niembro R A (1986) Mecanismos de Reproducción Sexual en Pinos. Ed. Limusa. México. $130 \mathrm{p}$

Núñez P A, R Demanet, T H Misselbrook, M Alfaro, M L Mora (2010) Nitrogen losses under different cattle grazing frequencies and intensities in a volcanic soil of southern Chile. Chilean J. Agric. Res. 70:237-250

Porta A, N Filliat, N Plata (1999) Phytotoxicity and phytoremediation studies in soils polluted by weathered oil. In: Phytoremediation and Innovative Strategies for Specialized Remedial Applications. B C Alleman, A Lesson (eds). Battell, Columbus. pp:51-56.
Pritchett W L (1991) Suelos Forestales: Propiedades, Conservación y Mejoramiento. Ed. Limusa. México, D. F. 634 p.

Quijada R M (1980) Ensayo de progenie. In: FAO, Mejora Genética de Árboles Forestales. Estudio FAO: Montes No. 20 Mérida, Venezuela. pp:224-230.

Ramírez H C, J J Vargas H, J López U (2005) Distribución y conservación de las poblaciones naturales de Pinus greggii. Acta Bot. Mex. 72:1-16.

Rodríguez F H, J Rodríguez A (2002) Métodos de Análisis de Suelos y Plantas. Criterios de Interpretación. Ed. Trillas. México, D. F. $196 \mathrm{p}$.

Rodríguez L R, S Valencia M, J Meza R, M A Capó A, A Reynoso P (2008) Crecimiento y características de la copa de procedencias de Pinus greggii Engelm. en Galeana, Nuevo León. Rev. Fitotec. Mex. 31:19-26.

SAS Institute (1987) Statistical Analysis System. SAS Institute Inc. Cary, NC, USA. $1028 \mathrm{p}$

Smithwick E H A, M G Turner, K L Metzger, T C Balser (2005) Variation in NH4+ mineralization and microbial communities with stand age in lodgepole pine (Pinus contorta) forests, Yellowstone National Park (USA). Soil Biol. Biochem. 37:1546-1559.

Vargas H J J, A Muñoz O (1988) Resistencia a sequía II. Crecimiento y supervivencia en plántulas de cuatro especies de Pinus. Agrociencia 72:197-208.

Zobel B, J Talbert (1988) Técnicas de Mejoramiento de Árboles Forestales. 1a ed. Ed. Limusa. México. 545 p. 